\title{
Possible Neuro-Sweet Disease Mimicking Brain Tumor in the Medulla Oblongata -Case Report-
}

\author{
Chihiro AKIBA, ${ }^{1}$ Takanori ESAKI, ${ }^{1}$ Maya ANDO, ${ }^{2}$ Tsuyoshi FURUYA, ${ }^{2}$ \\ Kazuyuki NODA,${ }^{2}$ Yasuaki NAKAO, ${ }^{1}$ Takuji YAMAMOTO ${ }^{1}$ \\ Yasuyuki OKUMA, ${ }^{2}$ and Kentaro MORI ${ }^{1}$ \\ Departments of ${ }^{1}$ Neurosurgery and ${ }^{2}$ Neurology, \\ Juntendo University Shizuoka Hospital, Izunokuni, Shizuoka
}

\begin{abstract}
A 62-year-old male presented with a rare case of possible neuro-Sweet Disease (NSD) mimicking brain tumor in the medulla oblongata, manifesting as numbness in the bilateral upper and lower extremities, gait disturbance, dysarthria, and swallowing disturbance which gradually deteriorated over 3 months. Magnetic resonance imaging showed a mass lesion in the medulla oblongata, extending to the upper cervical cord with rim enhancement by gadolinium. The preoperative diagnosis was brain tumor, such as glioma, or inflammatory disease. His neurological symptoms gradually deteriorated, so biopsy was performed through the midline suboccipital approach. Histological examination showed infiltration of inflammatory cells, mainly lymphocytes and macrophages. Human leukocyte antigen typing showed Cw1 and B54 which strongly suggested possible NSD. Steroid pulse therapy was started after surgery and the clinical symptoms improved. Neurosurgeons should be aware of inflammatory disorders such as NSD mimicking brain tumor.
\end{abstract}

Key words: neuro-Sweet disease, inflammatory disease, medulla oblongata, corticosteroid, brain biopsy

\section{Introduction}

Sweet disease is a multisystem inflammatory disorder, first described by Robert Douglas Sweet in $1964,{ }^{8)}$ manifesting as general fatigue, fever, leukocytosis, and erythematous plaques, ${ }^{4,5,8)}$ and characterized by painful erythematous plaques in the skin, and also in the various organs, including eyes, lungs, liver, kidneys, and gastrointestinal tract. Histological examination of the plaques shows

Received May 12, 2010; Accepted July 21, 2010 dense dermal infiltration of neutrophils without vasculitis. Sweet disease is considered to be an immunological disease because systemic administration of corticosteroids is effective, but antibiotics have no effect. Sweet disease is associated with preceding upper respiratory infection, pregnancy, inflammatory bowel disease, malignant diseases, and drug abuse. ${ }^{1)}$ Neuro-Sweet disease (NSD) was proposed as a distinct entity of encephalomeningitis in $1999,{ }^{4)}$ and the criteria for diagnosis of NSD were described in 2005.5) We previously reported two cases of NSD, but this disorder has not been well characterized. ${ }^{2,7)}$ 
Here, we report a rare case of possible NSD mimicking brain tumor in the medulla oblongata with histological findings obtained by brain biopsy.

\section{Case Report}

A 62-year-old male presented with numbness in the bilateral upper and lower extremities, gait disturbance, dysarthria, and swallowing disturbance which had gradually deteriorated over 3 months. The numbness started from the right upper extremity and extended to the left upper extremity, and sensory disturbance extended to the bilateral lower extremities. In addition, dysarthria and swallowing disturbance occurred. Positron emission tomography with L-methyl- ${ }^{11} \mathrm{C}$-methionine performed in another hospital indicated no evidence of tumor. He had a history of high blood pressure and he was taking medication. He had no history of preceding upper respiratory infection.

On admission, he was alert and well-orientated. Neurological examination revealed very slight left facial palsy, tongue protrusion to the right, absence of gag reflex bilaterally, dysarthria, swallowing disturbance, slight motor weakness in the right hand, dysesthesia in the bilateral forearms and planta, and decreased deep sensation in the right extremities. Romberg sign was positive and his gait was unsteady.

Laboratory examination showed white blood cell (WBC) count was slightly elevated to $8300 / \mathrm{mm}^{3}$ (normal range $4000-8000 / \mathrm{mm}^{3}$ ), but the demarcation of WBCs was normal. The serum C-reactive protein level was within the normal range. Cerebrospinal fluid (CSF) examination showed $1 \mathrm{cell} / \mathrm{mm}^{3}$ (mononuclear cells, 1; neutrophilic cells, 0), glucose concentration of $89 \mathrm{mg} / \mathrm{dl}$, and protein concentration of $84 \mathrm{mg} / \mathrm{dl}$. Culture of the CSF was negative for bacteria, tuberculosis, and fungi.

Computed tomography (CT) and magnetic resonance (MR) imaging demonstrated a mass lesion in the medulla oblongata, extending to the upper cervical cord. CT showed a low density area with slight enhancement by contrast medium (Fig. 1). MR imaging showed a hypo- to isointense area on $\mathrm{T}_{1}$-weighted imaging and a hyperintense area on both $\mathrm{T}_{2}$-weighted and fluid-attenuated inversion recovery (FLAIR) imaging. Gadolinium administration caused partial rim enhancement of the lesion (Fig. 2). Diffusion-weighted imaging showed no abnormality.

Biopsy was performed through the midline suboccipital approach with C1 laminectomy. The medulla was swollen but the color of the surface was normal. The posterior median sulcus was confirmed and the biopsy was performed in the right part of gracile fasciculus because the lesion extended to the right part of medulla and the upper cervical portion on MR imaging. He suffered no deterioration of symptoms after the operation. Histological examination showed infiltration of inflammatory cells, mainly lymphocytes and macrophages. Immunohistochemical staining for leukocyte common antigen was positive (Fig. 3). The differential diagnosis included inflammatory disease, such as meningitis, sarcoidosis, multiple sclerosis, and various collagen diseases or neuro-neutrophilic diseases. ${ }^{3)}$
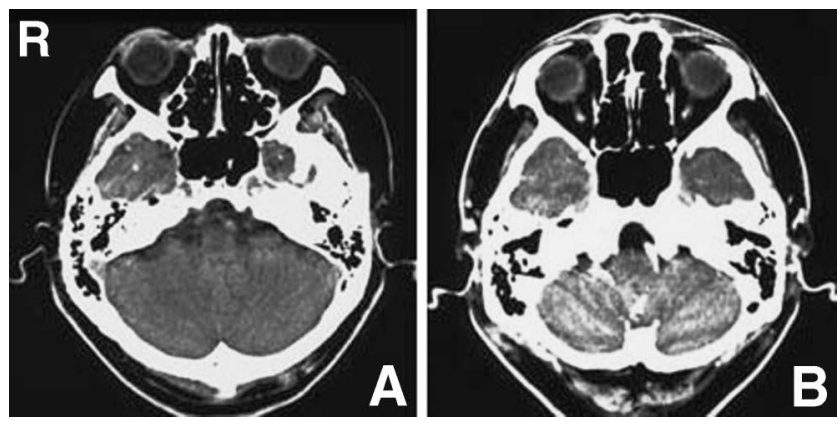

Fig. 1 Computed tomography scans showing a low density area in the medulla oblongata (A), with slight enhancement by contrast medium (B).
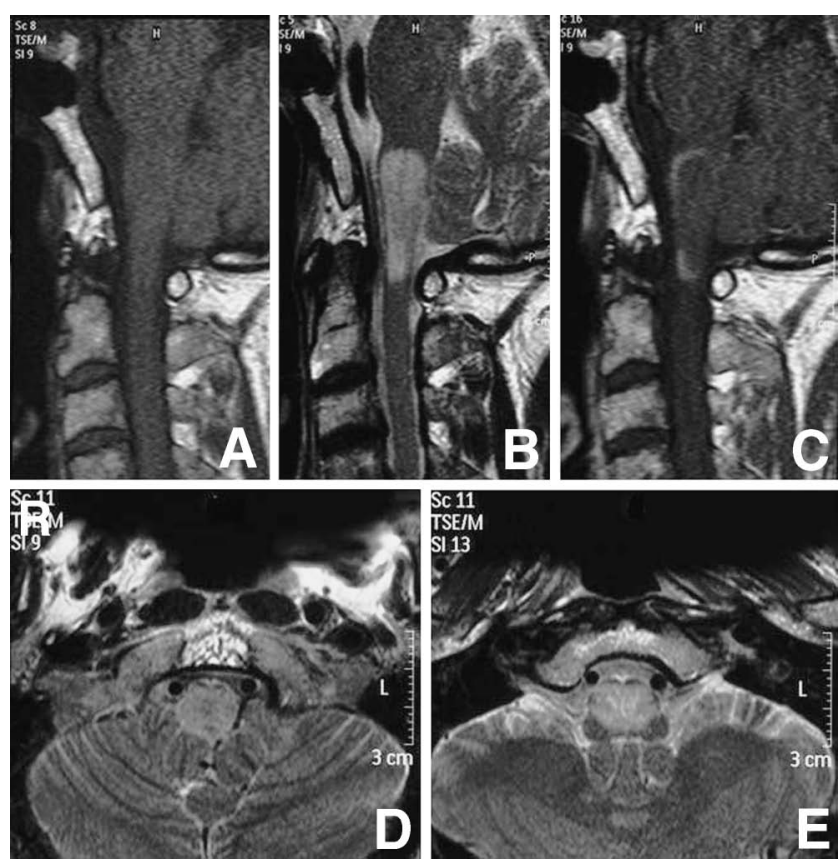

Fig. 2 Sagittal magnetic resonance images showing a hypo- to isointense mass on the $T_{1}$-weighted image (A), a hyperintense mass on the $T_{2}$-weighted image (B), and partial rim enhancement by gadolinium (C), located in the medulla oblongata and extending to the upper cervical portion. Axial $T_{2}$-weighted images showing a hyperintense mass in the medulla oblongata (D, E).

However, no specific findings for these disorders were obtained.

Various virus antibody values including Coxsackie virus, herpes simplex virus, human immunodeficiency virus, and syphilis or polymerase chain reaction of tubercle were all negative, so meningitis due to viruses or bacteria could be excluded. Serum lysozyme and angiotensin converting enzyme for the diagnosis of sarcoidosis, and oligoclonal band of CSF and serum anti-aquaporin 4 antibody for the diagnosis of multiple sclerosis and neuromyelitis optica were negative. Various autologous antibodies, including anti-nuclear antibody, anti-thyroid 
peroxidase antibody, anti-thyroglobulin antibody, antismooth muscle antibody, anti-SS-A/B antibody, and PR3/MPO-anti-neutrophil cytoplasmic antibody were also within the normal ranges. Human leukocyte antigen (HLA) typing showed B54 and Cw1, indicating the possibility of NSD. HLA-B51 for the diagnosis of neuroBehçet disease was negative. Dermatological and ophthalmologic examinations showed no skin lesion, or ocular lesions such as uveitis, episcleritis, and conjunctivitis. Whole-body CT with contrast medium did not demonstrate any lesions.

We suspected that the medullary lesion was associated
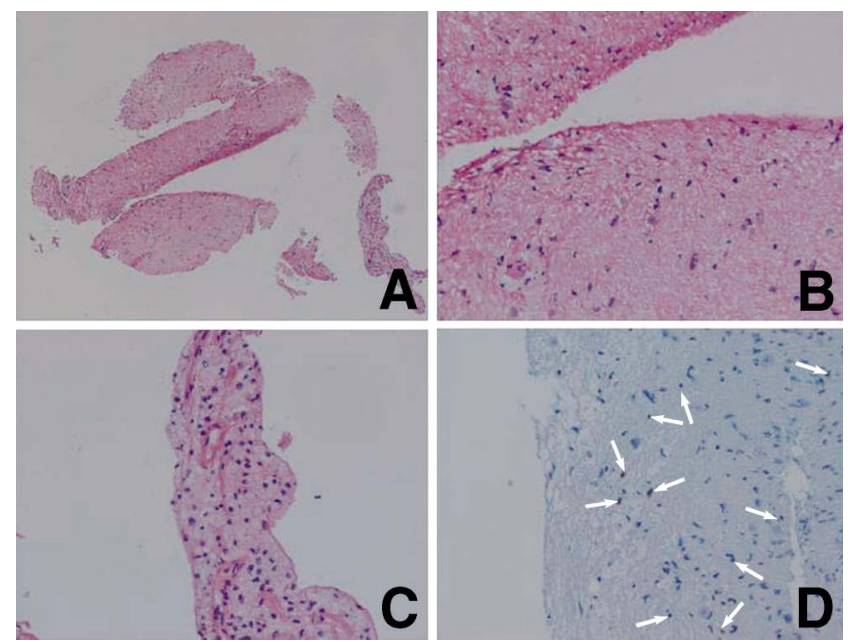

Fig. 3 A-C: Photomicrographs of biopsy specimens showing infiltration of inflammatory cells (A), mainly lymphocytes (B) and macrophages $(C)$ in the brain tissue. Hematoxylin and eosin stain, original magnification $A: \times 40$; $B$ and $C: \times 200$. D: Immunohistochemical staining for leukocyte common antigen with hematoxylin staining for nucleus showing that the cell membrane of lymphocytes stained in brown (arrows). Original magnification $\times 200$.
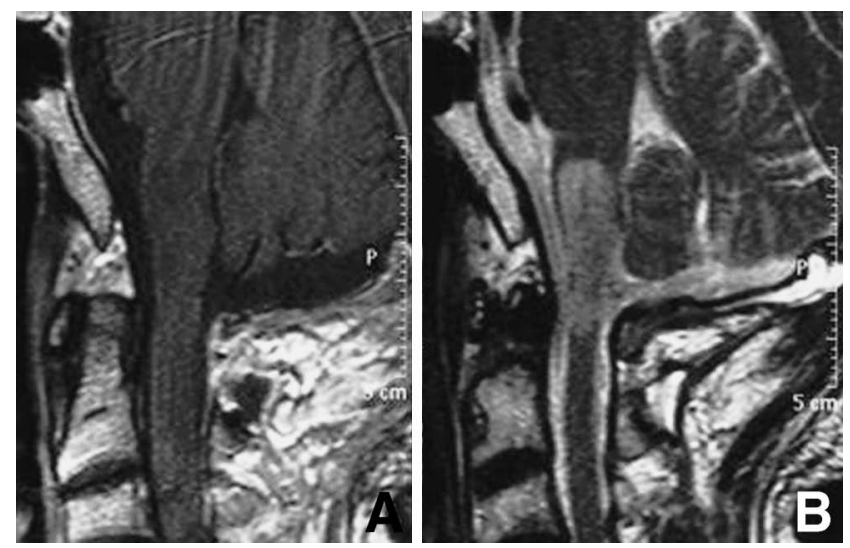

Fig. 4 Magnetic resonance images after steroid pulse therapy showing disappearance of enhancement on the $T_{1}$-weighted image with gadolinium (A), and a persistent hyperintense area on the $T_{2}$-weighted image (B). with NSD, so intravenous administration of methylprednisolone was initiated using $1000 \mathrm{mg} /$ day for 3 days followed by oral administration of prednisolone using 60 $\mathrm{mg} /$ day. After steroid pulse therapy, MR imaging showed disappearance of the enhancement with gadolinium (Fig. 4). His clinical symptoms also improved and he was discharged from our hospital. The steroid therapy has been tapered and he is now taking prednisolone $15 \mathrm{mg}$ daily under close observation as an outpatient of the neurological department.

\section{Discussion}

Clinical diagnostic criteria for NSD were proposed in 2005 as follows ${ }^{5}$ : 1) Neurological features, highly systemic response or sometimes spontaneous remission induced by glucocorticoid administration, and commonly recurrent encephalitis or meningitis, usually with fever over $38^{\circ} \mathrm{C} ; 2$ ) Dermatological features, painful or tender dull red erythematous plaques or nodules preferentially occurring on the face, neck, upper limbs, and upper part of the trunk, predominantly neutrophilic infiltration of the dermis, with spared epidermis and absence of leukocytoclastic vasculitis; 3) Other features, absence of cutaneous vasculitis and thrombosis, and absence of typical uveitis, which are all seen in Behçet disease; and 4) HLA association, HLA-Cw1 or B54 is positive, but HLA-B51 is negative. The diagnosis of probable NSD requires the presence of all criteria of 1), 2), and 3). The diagnosis of possible NSD requires any neurological manifestations, either criterion 2) or 4), and one item or more of 3) (Table 1).5)

Sweet disease shares common clinical features with Behçet disease, so a new entity, neuro-neutrophilic dis-

Table 1 Criteria for neuro-Sweet disease (NSD)

Our case

1) Neurological features

Highly systemic glucocorticoid responsive or sometimes spontaneously remitting, but frequently recurrent encephalitis or meningitis, usually with fever over $38^{\circ} \mathrm{C}$

2) Dermatological features

Painful or tender, dull red erythematous plaques or nodules preferentially occurring on the face, neck, upper limbs, and upper part of the trunk Predominantly neutrophilic infiltration of the dermis, spared epidermis, and absence of leukocytoclastic vasculitis

3) Other features

Absence of cutaneous vasculitis and thrombosis, which are seen in Behçet disease Absence of typical uveitis, which is seen in Behçet disease

4) HLA association

HLA-Cw1 or B54 positive

HLA-B51 negative

Probable NSD: all of 1), 2), and 3). Possible NSD: any neurological manifestations, either 2) or 4) and one item or more of 3). Any other neurological diseases that can explain the neurological symptoms and signs, except neuro-Behçet disease, should be excluded before the diagnosis of NSD is made. HLA: human leukocyte antigen. 
ease, has been proposed..$^{3-5)}$ NSD is characterized by neutrophilic infiltration without vasculitis, high responsiveness to systemic corticosteroid therapy, and association with HLA-Cw1 and B54. In contrast, neuro-Behçet disease is characterized by vasculitis and HLA-B51. The difference in ocular lesions characteristic of these diseases is also important for the differential diagnosis. Episcleritis and conjunctivitis are seen in Sweet disease, whereas uveitis is common in Behçet disease. However, our patient had no signs and symptoms of either NSD or neuro-Behçet disease. Only HLA-Cw1 and B54 suggested the possibility of NSD.

Neuroimaging demonstrates NSD as abnormal lesions on CT or MR imaging in various central nervous system regions without site predilection. Generally, the appearance is low density on CT, and hypointense on $\mathrm{T}_{1}$-weighted and hyperintense on $\mathrm{T}_{2}$-weighted or FLAIR MR imaging. Most lesions are not enhanced by contrast medium, but some lesions did show enhancement. ${ }^{5,9}$ The lesions may occur in any regions of the central nervous system, including the spinal cord. However, NSD in the brainstem is rare, especially in the lower part of the brainstem. ${ }^{9}$ Systemic steroid therapy resolves such abnormalities on CT or MR imaging in most cases. ${ }^{5)}$

The histological findings showed infiltration of inflammatory cells, mainly lymphocytes and macrophages. The infiltration of macrophages was similar to the previous findings from brain needle biopsy or autopsy in cases of NSD. ${ }^{5,6)}$ The specimens of the brain tissue were very small because the lesion was located in the medulla, so histological examination could not prove the absence of vasculitis and neutrophilic infiltration. Most previous diagnoses of NSD in the literature were based on biopsy of the skin lesion. However, our patient had no erythematous plaques.

Our patient fulfilled the criteria for possible NSD with neurological manifestations, positive HLA-Cw1 and B54, absence of typical uveitis, and good response to corticosteroid therapy (Table 1), although the possibility of other inflammatory diseases was not excluded. Because he had no dermatological lesion, the diagnosis of probable NSD was difficult to confirm. Although the biopsy was very small, it was useful for the diagnosis of inflammatory disease and excluding brain tumor, so we could start the corticosteroid pulse therapy with confidence.

The present rare case of possible NSD mimicking brain tumor in the medulla oblongata indicates that brain biopsy may be necessary in patients with atypical mass lesion, even if the lesion is located in the medulla. Neurosurgeons must be able to recognize neuro-inflammatory disorders such as NSD, which will promote successful management.

\section{References}

1) Cohen PR: Sweet's syndrome-a comprehensive review of an acute febrile neutrophilic dermatosis. Orphanet J Rare Dis 2: 34,2007

2) Fukae J, Noda K, Fujishima K, Wada R, Yoshiike T, Hattori N, Okuma Y: Successful treatment of relapsing neuro-Sweet's disease with corticosteroid and dapsone combination therapy. Clin Neurol Neurosurg 109: 910-913, 2007

3) Hisanaga K: Neuro-neutrophilic disease: neuro-Behçet disease and neuro-Sweet disease. Intern Med 46: 153-154, 2007

4) Hisanaga $\mathrm{K}$, Hosokawa M, Sato N, Mochizuki H, Itoyama $Y$, Iwasaki Y: "Neuro-sweet disease": benign recurrent encephalitis with neutrophilic dermatosis. Arch Neurol 56: 1010-1013, 1999

5) Hisanaga K, Iwasaki $Y$, Itoyama $Y$; Neuro-Sweet Disease Study Group: Neuro-Sweet disease: clinical manifestations and criteria for diagnosis. Neurology 64: 1756-1761, 2005

6) Kokubo Y, Kuzuhara S, Isoda K, Sato K, Kawada N, Narita Y: Neuro-Sweet disease: report of the first autopsy case. J Neurol Neurosurg Psychiatry 78: 997-1000, 2007

7) Noda K, Okuma Y, Fukae J, Fujishima K, Goto K, Sadamasa H, Yoshiike T, Mizuno Y: Sweet's syndrome associated with encephalitis. J Neurol Sci 188: 95-97, 2001

8) Sweet RD: An acute febrile neutrophilic dermatosis. Br J Dermatol 76: 349-356, 1964

9) Yamashita K, Shii H: [Imaging diagnosis and brainstem lesion of neuro-Sweet disease]. Shinkei Naika 64: 132-135, 2006 (Japanese)

Address reprint requests to: Takanori Esaki, MD, Department of Neurosurgery, Juntendo University Shizuoka Hospital, 1129 Nagaoka, Izunokuni, Shizuoka 410-2295, Japan. 